ARTICLE

Received 27 Mar 2015 | Accepted 14 Dec 2015 | Published 25 Jan 2016

DOI: $10.1038 /$ ncomms10459 OPEN

\title{
Persistent order due to transiently enhanced nesting in an electronically excited charge density wave
}

\author{
L. Rettig ${ }^{1,2, \dagger}$, R. Cortés ${ }^{2,3, \dagger}$, J.-H. Chu ${ }^{4,5}$, I.R. Fisher ${ }^{4,5}$, F. Schmitt ${ }^{4}$, R.G. Moore ${ }^{5}$, Z.-X. Shen ${ }^{4,5}$, P.S. Kirchmann ${ }^{5}$, \\ M. Wolf $2,3 \&$ U. Bovensiepen ${ }^{1,2}$
}

Non-equilibrium conditions may lead to novel properties of materials with broken symmetry ground states not accessible in equilibrium as vividly demonstrated by non-linearly driven mid-infrared active phonon excitation. Potential energy surfaces of electronically excited states also allow to direct nuclear motion, but relaxation of the excess energy typically excites fluctuations leading to a reduced or even vanishing order parameter as characterized by an electronic energy gap. Here, using femtosecond time- and angle-resolved photoemission spectroscopy, we demonstrate a tendency towards transient stabilization of a charge density wave after near-infrared excitation, counteracting the suppression of order in the non-equilibrium state. Analysis of the dynamic electronic structure reveals a remaining energy gap in a highly excited transient state. Our observation can be explained by a competition between fluctuations in the electronically excited state, which tend to reduce order, and transiently enhanced Fermi surface nesting stabilizing the order.

\footnotetext{
${ }^{1}$ Fakultät für Physik, Universität Duisburg-Essen, Lotharstr. 1, D-47057 Duisburg, Germany. ${ }^{2}$ Fachbereich Physik, Freie Universität Berlin, Arnimallee 14 , D-14195 Berlin, Germany. ${ }^{3}$ Abteilung Physikalische Chemie, Fritz-Haber-Institut der MPG, Faradayweg 4-6, D-14195 Berlin, Germany. ${ }^{4}$ Geballe Laboratory for Advanced Materials, Department of Applied Physics, Via Pueblo Mall, Stanford, California 94305, USA. ${ }^{5}$ SLAC National Accelerator Laboratory, Stanford Institute for Material and Energy Sciences, Menlo Park, 94025 California, USA. † Present address: Swiss Light Source, Paul Scherrer Institut, CH-5232 Villigen PSI, Switzerland (L.R.); Physical Review Letters, American Physical Society, 1 Research Road, Ridge, 11961 New York, USA (R.C.). Correspondence and requests for materials should be addressed to L.R. (email: laurenz.rettig@fu-berlin.de) or to U.B. (email: uwe.bovensiepen@uni-due.de).
} 
O rdered states in condensed matter emerge in thermodynamic equilibrium below a critical temperature $T_{\mathrm{c}}$ if competing thermal fluctuations are weak compared with the energy gain driving the order. This delicate balance depends critically on the dimensionality of a system, where lowdimensional structures introduce instabilities towards broken symmetry ground states, but on the same footing enhance fluctuations. Charge density waves (CDWs) form due to cooperative effects of electrons and the crystal lattice and represent widely studied model systems of such broken symmetry ground states. Under equilibrium conditions CDW formation can be described phenomenologically by effective mean field models ${ }^{1}$, but on a microscopic level a description is far from trivial, because the electronic and lattice contributions can hardly be disentangled due to their intrinsic coupling 2,3 . Optical excitation of CDW systems into non-equilibrium states faster than the characteristic time scales of electrons or phonons breaks this link and has opened opportunities to investigate the interactions underlying CDW formation ${ }^{4-11}$. In addition, tailored excitations of specific resonances in the mid-infrared have been reported to lead to novel, non-equilibrium material properties that are not accessible in thermal equilibrium ${ }^{12-16}$.

The formation of a CDW is driven by an instability of the electronic system to a spatially periodic perturbation. Particularly in quasi one-dimensional systems parallel parts of the Fermi surface (FS) are nested by an ordering vector $\boldsymbol{q}_{\mathrm{CDW}}$, and lead to a divergence of the electronic Lindhard susceptibility ${ }^{1}$. Electron-phonon (e-ph) coupling imprints this ordering tendency on the lattice and freezes a soft phonon mode into a periodic lattice distortion, leading to a complex many-body problem. While this is a widely considered explanation for CDW formation ${ }^{1,17}$, momentum-dependent e-ph coupling may modify this picture ${ }^{3,18,19}$. This coupled charge and lattice periodicity $1 / q_{\mathrm{CDW}}$ creates an energy gap $2 \Delta$ in the electronic structure due to Bragg scattering at the modulated charge density. Thereby the periodic lattice distortion and the CDW are stabilized by the strength of e-ph coupling and the diverging susceptibility, that is, the FS topology. Optical excitation creates fluctuations of the electron density, which on average reduce the electronic charge modulation and $2 \Delta$. Simultaneously, coherent lattice dynamics may drive the system towards a high-symmetry state and decrease the lattice distortion, further reducing the CDW modulation and $2 \Delta$. Such ultrafast suppression in $2 \Delta$ has been widely observed in previous experiments employing femtosecond time- and angle-resolved photoemission spectroscopy (trARPES) after femtosecond laser excitation ${ }^{5,6,20}$ in agreement with the theory ${ }^{21}$, which emphasizes the impact of optically induced fluctuations on the interaction leading to CDW formation. However, an enhanced driving force for CDW formation maintaining order in the non-equilibrium state was so far not observed.

Here we demonstrate a hindered melting of the CDW state after femtosecond laser excitation in the CDW model system $\mathrm{RTe}_{3}(\mathrm{R}=\mathrm{Dy}, \mathrm{Ho})$ through a residual gap $2 \Delta$ in a transient optically excited state. This residual $2 \Delta$ is fluence independent and is a consequence of improved FS nesting conditions in the excited state, for which we provide evidence through a complete analysis of $2 \Delta$ as a function of two independent momentum directions $k_{x}, k_{z}$, binding energy $E$ and pump-probe delay $t$. This improved nesting increases the interactions underlying CDW formation which compete with incoherent fluctuations induced by the excitation. This surprising observation suggests a pathway to control material properties under non-equilibrium conditions relevant for a wide range of material classes with broken symmetry ground states.

\section{Results}

Static FS of $\mathbf{R T e}_{3}$. We begin by analyzing the electronic structure of $\mathrm{HoTe}_{3}$ in thermal equilibrium using high-resolution laserARPES at $h v=7 \mathrm{eV}$, which is representative for the series of rareearth tritellurides. Its quasi-2D electronic structure is determined by square nets of Te-planes, which are stacked between buckled RTe layers that allow to tune the CDW temperature and gap size through chemical pressure ${ }^{22}$. Figure $1 \mathrm{~b}$ (left) shows the
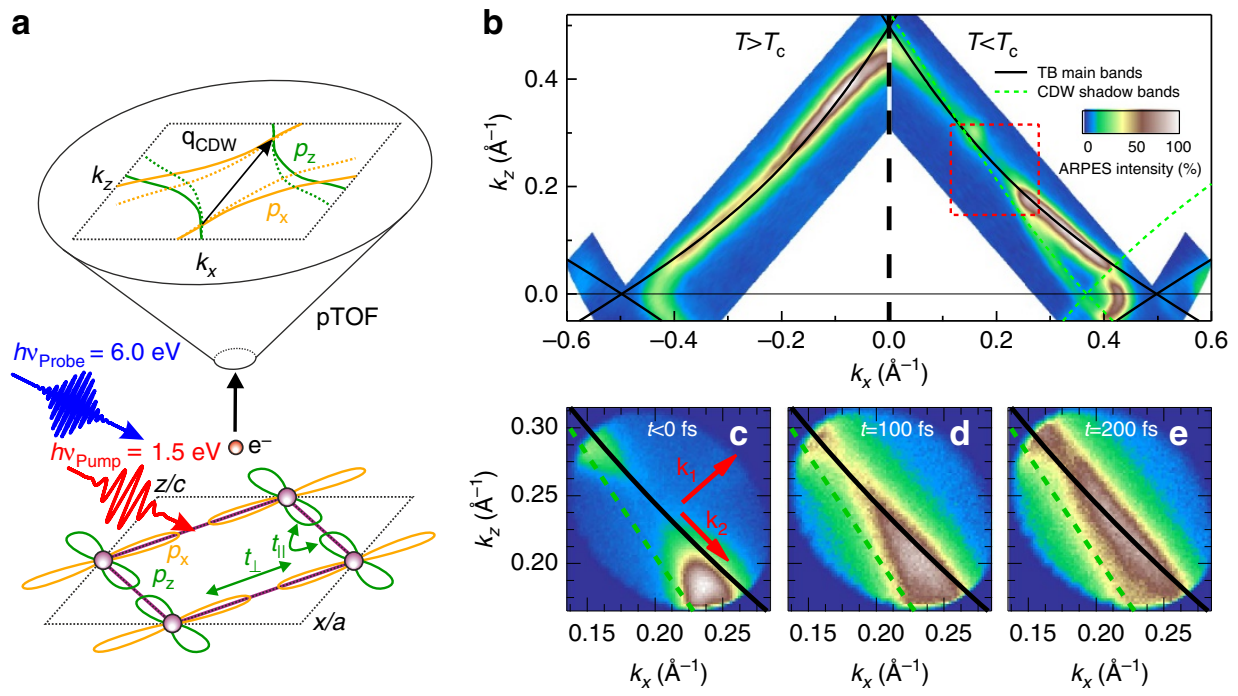

Figure 1 | Time-dependent Fermi surface map. (a) Experimental scheme and TB model. The Te- $5 p_{x / z}$ orbitals in the Te-layers of RTe 3 (bottom) are coupled by $t_{\|}$and $t_{\perp}$ describing interactions parallel and perpendicular to the Te chains and lead to a diamond-shaped Fermi surface in reciprocal space, which we excess by our position-sensitive time-of-flight photoelectron spectrometer (pTOF) (top). Nesting leads to shadow bands translated along $\mathbf{q}_{\mathrm{CDW}}$ (dashed lines). (b) Static FS of $\mathrm{HoTe}_{3}$ for $T=300 \mathrm{~K}>T_{\mathrm{c}}$ (left) and $T=180 \mathrm{~K}<T_{\mathrm{c}}$ (right). Main and shadow TB bands are indicated by solid and dashed lines, respectively. In the CDW phase, energy gaps open on the Fermi surface where main and shadow bands overlap. The red square indicates the region investigated in panels (c-e). (c-e) Time-dependent FS of DyTe ${ }_{3}$ for various pump-probe delays at $T<T_{\mathrm{c}}$. The gapped FS transforms within 200 fs into a continuous, metallic state. Arrows in $\mathbf{c}$ indicate directions perpendicular $\left(k_{1}\right)$ and parallel $\left(k_{2}\right)$ to the FS, respectively. The weak intensity in the upper left corner in c stems from a partially twinned crystal domain. 

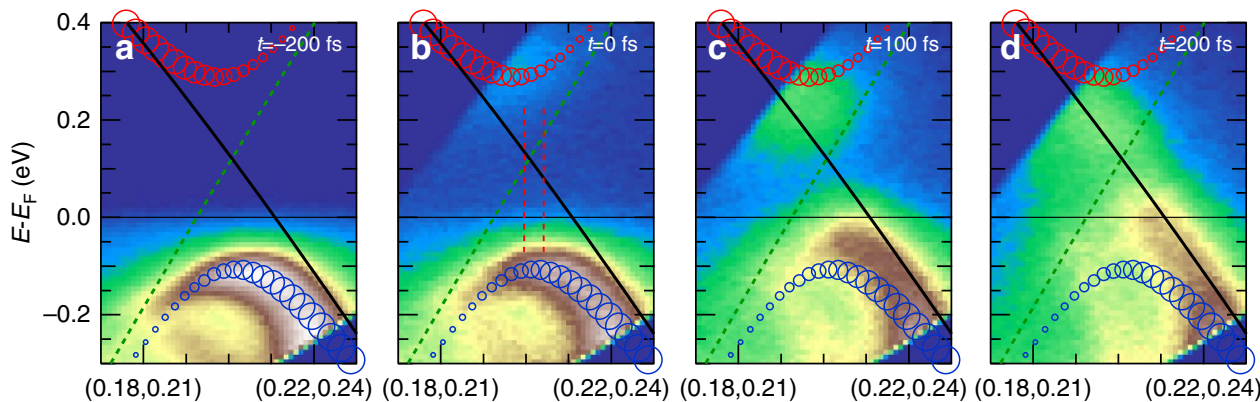

$\left(k_{x}, k_{z}\right)$ along $k_{1}\left(\AA^{-1}\right) \quad\left(k_{x}, k_{z}\right)$ along $k_{1}\left(\AA^{-1}\right)$

$\left(k_{x}, k_{z}\right)$ along $k_{1}\left(\AA^{-1}\right)$

$\left(k_{x}, k_{z}\right)$ along $k_{1}\left(\AA^{-1}\right)$
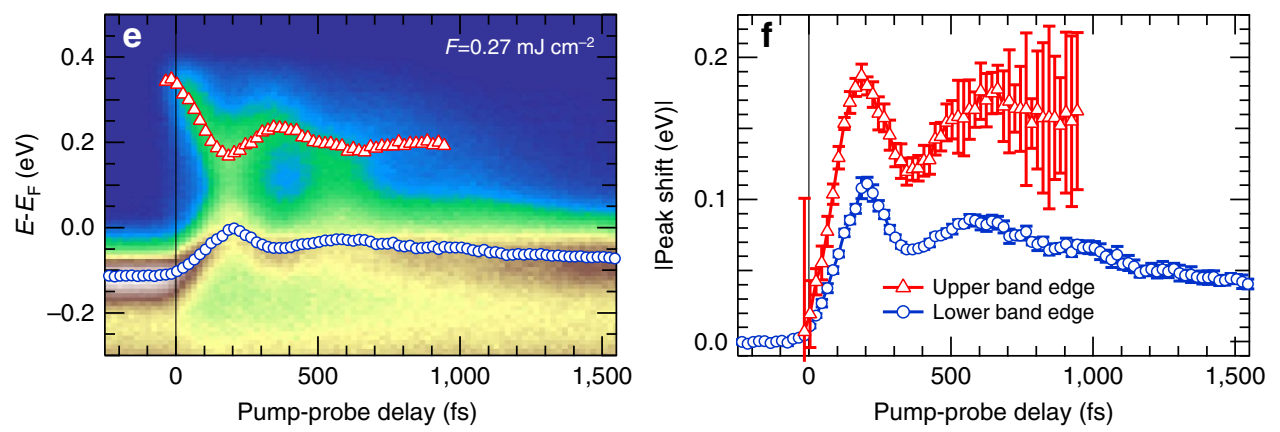

Figure 2 | Time-dependent CDW band structure. (a-d) trARPES data along $k_{1}$ for various pump-probe delays. Main and shadow TB bands are shown as solid and dashed lines, and symbols show lower (blue) and upper (red) band dispersion of the TB model including CDW gap, where the symbol sizes represent the spectral weight. (e) Spectra integrated around the region marked in $\mathbf{b}$ as a function of pump-probe delay. Red and blue symbols are peak positions of upper and lower CDW bands, respectively, obtained from a fitting procedure. (f) Peak shift of upper and lower band. Shown is the absolute value of the shift. Error bars represent $95 \%$ confidence intervals of the fits.

continuous, metallic $\mathrm{FS}$ of $\mathrm{HoTe}_{3}$ for $T>T_{\mathfrak{c}}$, which agrees well with tight binding (TB) calculations (solid lines) of the Te planes taking into account the overlap of $\mathrm{Te}-5 p_{x}$ and $5 p_{z}$ orbitals (refs 17,18), for details see Supplementary Note 1 and Supplementary Fig. 1. The curvature of the diamond-shaped FS in the $\left(k_{x}, k_{z}\right)$-plane is determined by the ratio of orbital overlap along and perpendicular to the chains of $p_{x} / p_{z}$-orbitals in the TB model described by $t_{\perp} / t_{\|}$(ref. 17), see Fig. 1a. For $T<T_{\mathrm{c}}$ (Fig. 1b, right) $\mathrm{CDW}$ formation results in shadow bands (green dashed lines), which are translated along $k_{z}$ by the nesting vector $\mathbf{q}_{\mathrm{CDW}} \approx 0.7 \mathrm{c}^{\star}$ (ref. 17) (Fig. 1a), and a CDW energy gap $2 \Delta$ opens in the vicinity of $\left(k_{x}, k_{z}\right)=(0.15,0.30) \AA^{-1}$ where the FS is nested due to overlapping main and shadow bands (see Supplementary Fig. 1). With decreasing $k_{z}$ the nesting gradually weakens and residual metallic pockets appear on the FS, where spectral weight is transferred into the shadow bands. Note that the imperfect nesting results in a $k$-dependent shift of the gap centre along the FS, while the full gap value $2 \Delta$ remains constant (see Supplementary Fig. 1). In the heavier members of $\mathrm{RTe}_{3}$, starting from $\mathrm{TbTe}_{3}$, also a second perpendicular CDW transition occurs along $a^{*}$ at a lower $T$ (refs 18,22). Here we concentrate on the first CDW transition with the larger $2 \Delta$, which is well separated on the FS.

Transient FS and band structure. Using femtosecond trARPES, see Fig. 1a, we investigate the electronic structure after fs laser excitation in $\mathrm{DyTe}_{3}$, which is very similar to $\mathrm{HoTe}_{3}$ (refs 17,18). Figure 1c-e shows the gapped FS region (red box in Fig. 1b) probed by $h v_{\text {probe }}=6.0 \mathrm{eV}$ after optical excitation with $h v_{\text {pump }}=1.5 \mathrm{eV}$ pump pulses at an absorbed fluence of $F=0.27$ $\mathrm{mJ} \mathrm{cm}{ }^{-2}$ for selected time delays. A movie of the transient FS is also available (Supplementary Movie 1). We observe filling of the gapped region starting from the metallic pocket. At $200 \mathrm{fs}$ a nearly ungapped FS is found, which is accompanied by a shift of spectral weight back to the TB main band. This provides direct evidence for an optically driven gap-closing transition. At a first glance the transient state at $200 \mathrm{fs}$ is very similar to the situation at $T>T_{\mathrm{c}}$ and consistent with the TB model ${ }^{6,23}$.

Our electron time-of-flight spectrometer ${ }^{24}$ allows extracting spectra along arbitrary in-plane momenta and we first analyze the trARPES intensity $I\left(E, k_{1}, t\right)$ with $k_{1}$ perpendicular to the FS contour, see Fig. 1c and Supplementary Movie 2. Figure 2 shows an occupied band with CDW gap before excitation. At $t=0$ fs the unoccupied band at the top of the CDW gap is populated, which enables a direct determination of the full transient $2 \Delta$. Both bands and the gap energy agree well with the interacting TB model (see Supplementary Note 1). Simultaneously, the occupied band is depleted, which implies hole excitation. With increasing pump-probe delay $t$ the lower and upper bands move towards each other as the CDW order recedes and $2 \Delta$ is reduced. At $t=100 \mathrm{fs}$ the lower band reaches $E_{\mathrm{F}}$ and at $t=200 \mathrm{fs}, E\left(k_{1}\right)$ is well described by the TB main band in agreement with the FS analysis above.

In Fig. 2e we plot trARPES spectra at $\mathbf{k}_{\mathrm{F}}$ and clearly observe periodic variations of $2 \Delta$. The peak positions of the lower and upper CDW bands are determined by Lorentzian line fits of $I(E, t)$, for details see Supplementary Note 2 and Supplementary Fig. 2. Before excitation, the lower CDW band occurs at $E-E_{\mathrm{F}}=-0.12(1) \mathrm{eV}$ (blue markers). Beginning at $t=0 \mathrm{fs}$, we monitor the upper $\mathrm{CDW}$ band at $E-E_{\mathrm{F}}=0.34(3) \mathrm{eV}$ (red markers) and determine $2 \Delta=0.46(4) \mathrm{eV}$ from the energy difference of the upper and lower CDW peaks. With increasing $t$ both lower and upper CDW bands shift towards $E_{\mathrm{F}}$. At $t=200 \mathrm{fs}$ the lower (upper) band reaches its maximum (minimum) and $2 \Delta$ is smallest before the bands shift away from $E_{\mathrm{F}}$ again at larger delays. Remarkably, two distinct peaks are observed in the spectra at all times (Supplementary Fig. 2), providing direct evidence for $2 \Delta$ remaining finite in the transient metallic state. 


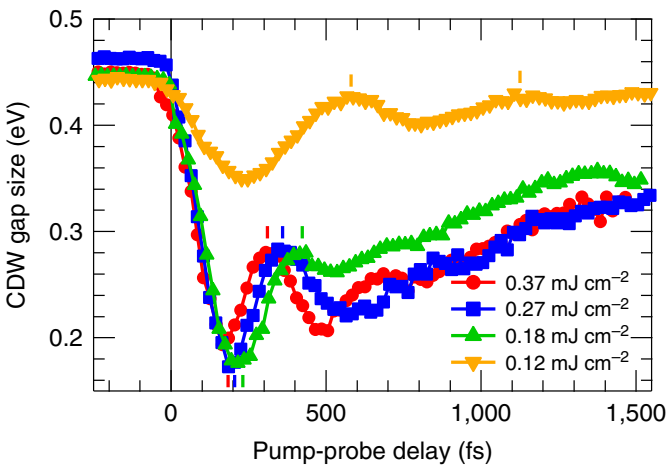

Figure 3 | Time-dependent CDW gap size. Time-dependent CDW gap size $2 \Delta$ determined from the separation of upper and lower CDW band as a function of pump-probe delay for various fluences.

Figure 3 shows $2 \Delta(t)$ (see Supplementary Note 3 and Supplementary Fig. 3) for various fluences $F$. For $F \leq 0.12 \mathrm{~mJ} \mathrm{~cm}^{-2}$ a relatively small decrease is followed by an oscillation with a period of $\sim 0.5 \mathrm{ps}$. This coherent response in a weakly perturbative regime lasts for several picoseconds and corresponds to the excitation of the CDW amplitude mode ${ }^{1,6,23,25-28}$. For higher fluence in a strongly perturbative regime $F \geq 0.18 \mathrm{~mJ} \mathrm{~cm}^{-2}$, corresponding to $\sim 220 \mathrm{meV}$ per unit cell (see Supplementary Note 4), which is comparable to the electronic energy gain upon $\mathrm{CDW}$ formation $\delta E \sim 250 \mathrm{meV}$ per unit cell estimated from the TB model (see Supplementary Note 1 and Supplementary Fig. 1), we find a stronger initial decrease of $2 \Delta$, followed by a recovery of $2 \Delta$ overlaid by anharmonic and strongly damped oscillations. These oscillations are a fingerprint of the coherent atomic rearrangements during the collapse and recovery of the $\mathrm{CDW}$ order in the transient potential energy surface. The minimal value of $2 \Delta$ saturates at $170 \mathrm{meV}$ independent of fluence for $F>0.12 \mathrm{~mJ} \mathrm{~cm}^{-2}$. This previously unobserved residual $2 \Delta$ in the transient metallic state demonstrates the persistence of electronic order in a highly perturbed system, in contrast to equilibrium conditions at $T>T_{\mathrm{c}}$ (ref. 18). This incomplete suppression of the CDW state is an unexpected result because at the highest fluence an excess energy of almost twice the energy gained by gapping the electronic structure is injected and still the gap persists (Fig. 3).

Asymmetric gap suppression. Our experimental approach further allows for the analysis of the symmetry of $2 \Delta$. A closer look at the transient peak positions in Fig. 2e reveals a larger pump-induced shift of the upper than of the lower CDW peak, indicating an asymmetric decrease of $2 \Delta$. From the relative shift of both bands in Fig. $2 \mathrm{f}$ we find a maximal shift of $110 \mathrm{meV}$ for the lower band (blue), while the upper band (red) changes by $60 \%$ more up to $190 \mathrm{meV}$. The momentum dependence of $I\left(E, k_{2}, t\right)$ along $k_{2}$ (see Fig. 1c) parallel to the FS contour is shown in Figs 4a,b for $t=0$ and $200 \mathrm{fs}$ and exhibits lower and upper CDW bands dispersing along $k_{2}$. A full movie is available as Supplementary Movie 3. This relates to a shift of the centre of $2 \Delta$ along the FS because $2 \Delta(\mathbf{k})$ is only centred around $E_{\mathrm{F}}$ for perfect nesting ${ }^{17}$ near $\left(k_{x}, k_{z}\right)=(0.17,0.29) \AA^{-1}$. To study the transient state, it is helpful to look at the pump-induced changes where the gap is minimal ( $t=200 \mathrm{fs})$. The pump-induced peak shifts (see Supplementary Note 5 and Supplementary Fig. 4) $\left|E\left(k_{2}\right)^{\text {up,down }}(200 \mathrm{fs})-E\left(k_{2}\right)^{\text {up,down }}(0 \mathrm{fs})\right|$ are depicted in Fig. 4c and exhibit a pronounced dependence on the FS position: With decreasing $k_{x}$, the transient shift of the upper band decreases, while the shift of the lower band increases, leading to a smaller asymmetry of the gap reduction. Finally, at $\left(k_{x}, k_{z}\right) \sim(0.17$,
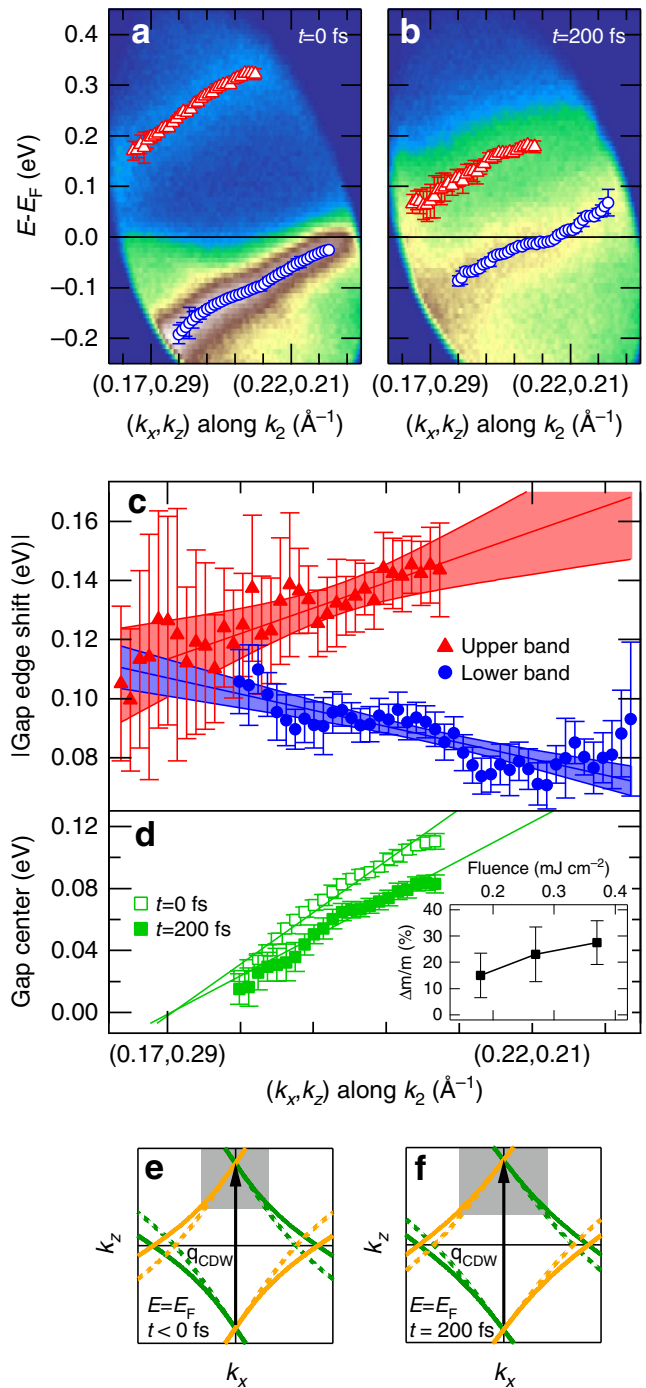

Figure 4 | Gap collapse along the Fermi surface. (a,b) Electron dispersion along $k_{2}$ for $t=0 \mathrm{fs}$ and $t=200 \mathrm{fs}$, respectively. Markers denote energy positions of upper (red) and lower (blue) CDW bands. (c) Peak shift between $t=0 \mathrm{fs}$ and $=200 \mathrm{fs}$ of upper and lower CDW band as function of position on the FS determined by fits, with error bars as $95 \%$ confidence intervals. Lines and shaded areas are linear fits and 95\% confidence bands, respectively. (d) Centre of the CDW gap as a function of position on the FS at $t=0 \mathrm{fs}$ (open symbols) and at $t=200 \mathrm{fs}$ (filled symbols). Solid lines are linear fits. The inset shows the relative change of slope $\Delta \mathrm{m} / \mathrm{m}$ as function of fluence. (e,f) Tight binding bands for $t_{\perp}=0.35 \mathrm{eV}(\mathbf{e})$ and $t_{\perp}=0.25 \mathrm{eV}(\mathbf{f})$. The grey areas marks the regions of good nesting where main (solid) and shadow (dashed) band lines overlap.

0.29) $\AA^{-1}$ both peak shifts become equal and the CDW gap decreases symmetrically around its centre. These observations manifest in a transient reduction of the dispersion of the gap centre with $k_{2}$, as seen in Fig. $4 \mathrm{~d}$.

\section{Discussion}

Such a transient change of the gap centre indicates an ultrafast modification of the nesting condition. The nesting vector and the curvature of the bands (Fig. 1) determine the band crossing of main and shadow bands (Fig. 2) and thus $2 \Delta(\mathbf{k})$. Changes of $q_{\mathrm{CDW}}$ alone can be ruled out to be driving an asymmetric closing of $2 \Delta$ since this would lead to a constant shift along the FS (see Supplementary Note 6 and Supplementary Fig. 5) and since 
time-resolved diffraction ${ }^{29}$ does not resolve such changes. In contrast, a transient modification of the electronic band dispersion, which can be parametrized with modified TB parameters, can explain the observed modification of the gap dispersion (Supplementary Fig. 5). The experimentally observed smaller gap dispersion can be reproduced by a reduction of $t_{\perp}$ leading to a smaller curvature of the TB FS, illustrated in Figs $4 \mathrm{e}, \mathrm{f}$. This results in a slower deviation from perfect nesting of main and shadow bands along the FS, and hence an improved nesting, as shown by the increase of the grey area in Fig. $4 \mathrm{f}$. In the limit of a vanishing curvature, the whole FS would be nested (we note that to maintain nesting for large changes of $t_{\perp}$, also a slight change of $q_{\mathrm{CDW}}$ is required). To capture the experimental change in gap dispersion a reduction of $t_{\perp}$ as large as $25 \%$ in the TB model is necessary (see Supplementary Note 7 and Supplementary Fig. 6). This scenario also explains the symmetric gap reduction at the point of perfect nesting $\left(\left(k_{x}, k_{z}\right) \sim(0.17,0.29) \AA^{-1}\right)$ where $2 \Delta$ is symmetric to $E_{\mathrm{F}}$. We conclude to observe a trend towards enhanced nesting, which was not observed as a function of temperature ${ }^{17,18}$ and must be unique for non-equilibrium conditions.

The gap dynamics is linked to ion motion, as evidenced by the coherent oscillations of $2 \Delta$. We speculate that such directed ion motion in the transient potential of the CDW distortion could transiently modify the orbital overlap in the network of Te orbitals and lead to the observed modification of the electronic dispersions and the improved FS nesting concluded above. As such, the enhanced nesting stabilizes the CDW in the optically excited state, however, in severe competition with fluctuations due to the excess energy, which destabilizes the CDW. The dynamics of the transient CDW state are therefore governed by the balance of these competing contributions. The limit in reduction of $2 \Delta \geq 0.17 \mathrm{eV}$ observed in Fig. 3 originates from compensation between the increased fluctuations and the enhanced nesting, with their respective destabilizing and stabilizing effects on the CDW. While increasing fluctuations are the direct consequence of pumping at larger $F$, the stabilizing tendency is evidenced by the increasingly improved nesting for higher $F$ (inset of Fig. $4 \mathrm{~d}$ ).

The possibility of modifying the transient electronic dispersion by optical excitation promises future control pathways for transient order in broken symmetry ground states of quantum materials as a complementary approach to nonlinear phononics ${ }^{12,15}$. Optimization of the excitation conditions may lead to desired ordered states, which requires modifications of the competing contributions towards stabilization and destabilization of the ordered state. Such a controlled approach might well be possible by adjusting pump photon energy and intensity since a reduction of photon energy results in lower excitation probability of secondary processes and related fluctuations while intensity defines the instantaneous excited state potential.

\section{Methods \\ Static high-resolution ARPES measurements. Single crystals of $\mathrm{RTe}_{3}$ were} grown by slow cooling of a binary melt ${ }^{22}$ and cleaved in ultrahigh vacuum (base pressure $<7 \times 10^{-11}$ mbar $)$ at $T=10 \mathrm{~K}$. Static FS maps of $\mathrm{HoTe}_{3}\left(T_{\mathrm{cl}}=285 \mathrm{~K}\right.$, $\left.T_{\mathrm{c} 2}=120 \mathrm{~K}\right)$ at $T=180 \mathrm{~K}$ and $T=300 \mathrm{~K}$ have been obtained using a laser-based ARPES setup with $h v=7 \mathrm{eV}$ and using a hemispherical electron analyzer (Scienta SES2000) (ref. 30). Energy and momentum resolution were better than $5 \mathrm{meV}$ and $0.005 \AA^{-1}$, respectively.

Time-resolved ARPES measurements. For the trARPES experiments, the output of a commercial amplified Ti:sapphire laser system (Coherent RegA 9050) operating at $300 \mathrm{kHz}$ repetition rate was used. Part of its output was frequencyquadrupled to yield $h v=6.0 \mathrm{eV}$ and used as probe pulses, while another part of the fundamental beam was time-delayed for excitation of the DyTe ${ }_{3}\left(T_{\mathrm{cl}}=305 \mathrm{~K}\right.$, $T_{\mathrm{c} 2}=50 \mathrm{~K}$ ) sample, held at $T=30 \mathrm{~K}$ during the measurements. The sample was mounted on a $45^{\circ}$ slanted sample holder and oriented by Laue diffraction before
trARPES measurements, which is necessary to reach the FS of $\mathrm{RTe}_{3}$ at a typical kinetic energy of $0.9 \mathrm{eV}$ and large emission angles of $>50^{\circ}$.

Position-sensitive time-of-flight spectrometer. A self-built position-sensitive time-of-flight spectrometer ${ }^{24}$ was used for photoelectron detection, mounted inside an ultrahigh vacuum chamber with a base pressure of $<7 \times 10^{-11} \mathrm{mbar}$. It enables simultaneous access to both in-plane momentum components $k_{x}$ and $k_{z}$, along with the kinetic energy of the electrons, and thus allows efficient mapping of the electronic band structure. It consists of a field-free drift tube for photoelectrons, combined with a micro channel plate and two-dimensional delay line detector (RoentDek Hexanode Hex80). From the arrival time and impact position on the micro channel plate, the electron kinetic energy $E_{\text {kin }}$ and both in-plane momentum components $k_{x}, k_{z}$ of each single photoelectron are calculated and stored in a threedimensional $\left(E, k_{x}, k_{z}\right)$ grid $^{24}$. The Hexanode design of the detector allows for efficient detection of multiple electrons per laser pulse, which is a prerequisite for obtaining high-quality trARPES data as presented in this work at typical count rates of $\sim 150 \mathrm{kHz}$.

The overall temporal, spectral and momentum resolutions of the setup were $100 \mathrm{fs}, 50 \mathrm{meV}$ and $2 \times 10^{-3} \AA^{-1}$, respectively. For further details of the experimental setup see Schmitt et al. ${ }^{6,23}$.

\section{References}

1. Grüner, G. in Density Waves in Solids, ed. Pines, D. (Addison-Wesley, Reading, MA, 1994).

2. Rossnagel, K. On the origin of charge-density waves in select layered transition metal dichalcogenides. J. Phys. Condens. Matter 23, 213001 (2011).

3. Eiter, H.-M. et al. Alternative route to charge density wave formation in multiband systems. Proc. Natl Acad. Sci. USA 110, 64-69 (2013).

4. Demsar, J., Biljakovic, K. \& Mihailovic, D. Single particle and collective excitations in the one-dimensional charge density wave solid $\mathrm{K}_{0.3} \mathrm{MoO}_{3}$ probed in real time by femtosecond spectroscopy. Phys. Rev. Lett. 83, 800-803 (1999).

5. Perfetti, L. et al. Time evolution of the electronic structure of $1 \mathrm{~T}-\mathrm{TaS}_{2}$ through the insulator-metal transition. Phys. Rev. Lett. 97, 067402 (2006).

6. Schmitt, F. et al. Transient electronic structure and melting of a charge density wave in $\mathrm{TbTe}_{3}$. Science 321, 1649-1652 (2008).

7. Eichberger, M. et al. Snapshots of cooperative atomic motions in the optical suppression of charge density waves. Nature 468, 799-802 (2010).

8. Rohwer, T. et al. Collapse of long-range charge order tracked by time-resolved photoemission at high momenta. Nature 471, 490-493 (2011).

9. Petersen, J. C. et al. Clocking the melting transition of charge and lattice order in $1 T-\mathrm{TaS}_{2}$ with ultrafast extreme-ultraviolet angle-resolved photoemission spectroscopy. Phys. Rev. Lett. 107, 177402 (2011).

10. Huber, T. et al. Coherent structural dynamics of a prototypical charge-densitywave-to-metal transition. Phys. Rev. Lett. 113, 026401 (2014).

11. Porer, M. et al. Non-thermal separation of electronic and structural orders in a persisting charge density wave. Nat. Mater. 13, 857-861 (2014).

12. Forst, M. et al. Nonlinear phononics as an ultrafast route to lattice control. Nat Phys. 7, 854-856 (2011).

13. Fausti, D. et al. Light-induced superconductivity in a stripe-ordered cuprate. Science 331, 189-191 (2011).

14. Kim, K. W. et al. Ultrafast transient generation of spin-density-wave order in the normal state of $\mathrm{BaFe}_{2} \mathrm{As}_{2}$ driven by coherent lattice vibrations. Nat. Mater 11, 497-501 (2012).

15. Mankowsky, R. et al. Nonlinear lattice dynamics as a basis for enhanced superconductivity in $\mathrm{YBa}_{2} \mathrm{Cu}_{3} \mathrm{O}_{6.5}$. Nature 516, 71-73 (2014).

16. Stojchevska, L. et al. Ultrafast switching to a stable hidden quantum state in an electronic crystal. Science 344, 177-180 (2014).

17. Brouet, V. et al. Angle-resolved photoemission study of the evolution of band structure and charge density wave properties in $\mathrm{RTe}_{3}(\mathrm{R}=\mathrm{Y}, \mathrm{La}, \mathrm{Ce}, \mathrm{Sm}, \mathrm{Gd}$, Tb, and Dy). Phys. Rev. B 77, 235104 (2008).

18. Moore, R. G. et al. Fermi surface evolution across multiple charge density wave transitions in $\mathrm{ErTe}_{3}$. Phys. Rev. B 81, 073102 (2010).

19. Maschek, M. et al. Wave-vector-dependent electron-phonon coupling and the charge-density-wave transition in $\mathrm{TbTe}_{3}$. Phys. Rev. B 91, 235146 (2015)

20. Hellmann, S. et al. Time-domain classification of charge-density-wave insulators. Nat. Commun. 3, 1069 (2012).

21. Shen, W. et al. Nonequilibrium 'Melting' of a charge density wave insulator via an ultrafast laser pulse. Phys. Rev. Lett. 112, 176404 (2014).

22. Ru, N. et al. Effect of chemical pressure on the charge density wave transition in rare-earth tritellurides $\mathrm{RTe}_{3}$. Phys. Rev. B 77, 035114 (2008).

23. Schmitt, F. et al. Ultrafast electron dynamics in the charge density wave material $\mathrm{TbTe}_{3}$. New J. Phys. 13, 063022 (2011).

24. Kirchmann, P. S. et al. A time-of-flight spectrometer for angle-resolved detection of low energy electrons in two dimensions. Appl. Phys. A 91, 211-217 (2008).

25. Yusupov, R. V., Mertelj, T., Chu, J.-H., Fisher, I. R. \& Mihailovic, D. Singleparticle and collective mode couplings associated with 1- and 2-directional 
electronic ordering in metallic $\mathrm{RTe}_{3}(\mathrm{R}=\mathrm{Ho}, \mathrm{Dy}, \mathrm{Tb})$. Phys. Rev. Lett. 101, 246402 (2008).

26. Yusupov, R. et al. Coherent dynamics of macroscopic electronic order through a symmetry breaking transition. Nat. Phys. 6, 681-684 (2010).

27. Rettig, L., Chu, J.-H., Fisher, I. R., Bovensiepen, U. \& Wolf, M. Coherent dynamics of the charge density wave gap in tritellurides. Faraday Discuss. 171, 299-310 (2014).

28. Leuenberger, D. et al. Classification of collective modes in a charge density wave by momentum-dependent modulation of the electronic band structure. Phys. Rev. B 91, 201106 (2015).

29. Han, T.-R. T. et al. Structural dynamics of two-dimensional charge-density waves in $\mathrm{CeTe}_{3}$ investigated by ultrafast electron crystallography. Phys. Rev. B 86, 075145 (2012).

30. Vishik, I. M. et al. Doping-dependent nodal fermi velocity of the high-temperature superconductor $\mathrm{Bi}_{2} \mathrm{Sr}_{2} \mathrm{CaCu}_{2} \mathrm{O}_{8+\delta}$ revealed using high-resolution angle-resolved photoemission spectroscopy. Phys. Rev. Lett. 104, $207002(2010)$

\section{Acknowledgements}

We acknowledge support from the Deutsche Forschungsgemeinschaft through BO 1823/2, SFB 616 and FOR 1700, and from the US Department of Energy, Office of Basic Energy Sciences under contract DE-AC02-76SF00515. R.C. acknowledges the AvH Foundation.

\section{Author contributions}

L.R. and R.C. carried out the experiments with support from F.S.; J.H. C. and I.R.F. provided the samples; L.R. analyzed the data with support from R.G.M.; L.R., P.S.K. and U.B. wrote the manuscript. U. B.,Z.-X.S. and M.W. designed the experiment; all author commented on the paper.

\section{Additional information}

Supplementary Information accompanies this paper at http://www.nature.com/ naturecommunications

Competing financial interests: The authors declare no competing financial interests. Reprints and permission information is available online at http://npg.nature.com/ reprintsandpermissions/

How to cite this article: Rettig, L. et al. Persistent order due to transiently enhanced nesting in an electronically excited charge density wave. Nat. Commun. 7:10459 doi: 10.1038/ncomms10459 (2016)

\section{(c) (1)}

This work is licensed under a Creative Commons Attribution 4.0 International License. The images or other third party material in this article are included in the article's Creative Commons license, unless indicated otherwise in the credit line; if the material is not included under the Creative Commons license, users will need to obtain permission from the license holder to reproduce the material. To view a copy of this license, visit http://creativecommons.org/licenses/by/4.0/ 